\title{
On the Differential Fractional Transformation Method of MSEIR Epidemic Model
}

\author{
Hanaa Abdelhamed Asfour \\ Mathematics Department, \\ Faculty of Eduction, \\ Ain Shams University, \\ Cairo, Egypt
}

\author{
Mohamed Ibrahim \\ Mathematics Department, \\ Faculty of Eduction, \\ Cairo University, \\ Cairo, Egypt
}

\begin{abstract}
In this paper we solve the MSEIR epidemic model by using the differential fractional transformation method. Using the differential Riemann-Liouville and the Caputo fractional derivative; we study convergent of MSEIR epidemic model; we use some theorems of fractional to introduce the solution of MSEIR epidemic Model. Numerical results are provided to confirm the theoretical result and the efficiency of the proposed method.
\end{abstract}

\section{Keywords}

Caputo and Riemann-Liouville of fractional; theorems of fractional; we study convergent of MSEIR epidemic Model; MSEIR Model; numerical solutions

\section{INTRODUCTION}

We consider some numerical aspects of the well-known MSEIR model, and note that this short paper can be viewed either as a companion of or on its own. The MSEIR model is a compartmental model for the spread of a disease in a population of size $N(T)$, which is divided into five epidemiological classes: passive immune newborns $M$; susceptible (non-immune) $S$; exposed $E$; infective $I$; and resistant (or immune) R.

Nonlinear equations: ordinary differential equations (ODEs) and partial differential equations (PDEs) are difficult to solve than linear especially by means of analytic methods. Traditionally, perturbation and asymptotic techniques are widely applied to obtain analytic approximations of nonlinear problems in science, engineering, finance and do great contribution to help us understand many nonlinear phenomena. However, it is well known that perturbation methods are strongly dependent upon small/large physical parameters, and therefore are valid in principle only for weakly nonlinear problems.

The so-called non-perturbation techniques, such as the Lyapunov's artificial small parameter method, the $\delta$ expansion method, a domain's decomposition method, and so on are formally dependent of small/large physical parameters. But all of these traditional non-perturbation methods cannot ensure the convergence of solution series: they are in fact only valid for weakly nonlinear problems, too. (Liao, 2004).

If idon (2009) used Homotopy analysis to the solution of the steady flow of a viscous incompressible fluid past a fixed circular cylinder. He calculated drag coefficients at 6th-order approximation and found to agree reasonably well with experimental measurements.

Liao (2004), in his paper described the basic ideas and current developments of the Homotopy analysis method. He also discussed some open questions and a hypothesis is put forward for future studies. Vahdatis (2013) also considered the spread of a non-fatal disease in a population which is assumed to have constant size over the period of the epidemic.

The system of differential equations for the numbers in the epidemiology classes and the population size is [20].

$$
\begin{aligned}
& \frac{d M}{d t}=b(\mathrm{~N}-\mathrm{S})-(\delta+d) M, \\
& \frac{d S}{d t}=b S+\delta M-\frac{\beta S I}{N}-\mathrm{dS}, \\
& \frac{d E}{d t}=\frac{\beta S I}{N}-(\varepsilon+d) \mathrm{E}, \\
& \frac{d I}{d t}=\varepsilon E-(\gamma+d) \mathrm{I}, \\
& \frac{d R}{d t}=\gamma R-d \mathrm{R}, \\
& \frac{d N}{d t}=(\mathrm{b}-\mathrm{dN}) .
\end{aligned}
$$

Using the differential equation for $\mathrm{N}$, eliminating the differential equation for $\mathrm{s}$ by using $m=1-s-e-i-r, b=d+q$ and $\lambda=\beta i$ Hethcote (2000). Then the differential equations for the MSEIR model are:

$$
\begin{aligned}
& \frac{d M}{d t}=(d+q)(\mathrm{E}+I+R)-\delta M, \\
& \frac{d S}{d t}=-\beta S I+\delta M, \\
& \frac{d E}{d t}=\beta S I-(\varepsilon+d+q) \mathrm{E}, \\
& \frac{d I}{d t}=\varepsilon E-(\gamma+d+q) \mathrm{I}, \\
& \frac{d R}{d t}=\gamma R-(d+q) \mathrm{R},
\end{aligned}
$$

With initial condition

$$
\begin{aligned}
& \mathrm{M}(0)=M_{0}, \mathrm{~S}(0)=S_{0}, \mathrm{E}(0)=E_{0}, \text { and } \\
& I(0)=I_{0}, R(0)=R_{0} .
\end{aligned}
$$


Such that $M+S+E+I+R=1$. The parameters $d, q, \gamma, \beta, \delta$ and $\varepsilon$ are positive real numbers.

Recently great considerations have been made to the models of FDEs in different area of researches. The most essential property of these models is their nonlocal property which does not exist in the integer order differential operators. We mean by this property that the next state of a model depends not only upon its current state but also upon all of its historical states. Now we introduce fractional order into the ODE model.

$$
\begin{aligned}
& D^{\alpha_{1}} m=(d+q)(\mathrm{e}+\mathrm{i}+\mathrm{r})-\delta \mathrm{m} \\
& D^{\alpha_{2}} s=-\beta s i+\delta \mathrm{m} \\
& D^{\alpha_{3}} e=\beta s i-(\varepsilon+d+q) e \\
& D^{\alpha_{4}} i=\varepsilon e-(\gamma+d+q) i \\
& D^{\alpha_{5}} r=\gamma i-(d+q) r
\end{aligned}
$$

$$
D_{R}^{\alpha} f(x)=\left\{\begin{array}{l}
\frac{1}{\Gamma(\mathrm{m}-\alpha)} \frac{d^{m}}{d x^{m}} \int_{0}^{x_{0}} \frac{\mathrm{f}(t)}{(x-t)^{\alpha-m+1}} d t \\
\frac{d^{m} f(x)}{d x^{m}}
\end{array}\right.
$$

Definition 2: The Caputo fraction derivative operator $D_{R}^{\alpha}$ of order $\alpha$ is defined in the following form.

$$
D^{\alpha} f(x)=\frac{1}{\Gamma(m-\alpha)} \int_{0}^{x} \frac{f^{m}(t)}{(x-t)^{\alpha-m+1}} d t, \quad \alpha>0
$$

Where $m-1<\alpha<m, \quad m \in \square$

The following theorem that are given below, for proofs and details see [19]

Theorems of fraction $[16,17]$ :

Theorem 1:

$$
\begin{aligned}
& \text { If } f(x)=g(x) \pm h(x), \text { then } \\
& F(\mathrm{k})=\mathrm{G}(\mathrm{k}) \pm H(k)
\end{aligned}
$$

$$
\begin{gathered}
\text { If } f(x)=g(x) h(x), \text { then } \\
F(k)=\sum_{l=0}^{k} G(l) H(k-l)
\end{gathered}
$$

Theorem 3:
With initial conditions

$$
\begin{aligned}
& \mathrm{M}(0)=\mathrm{m}_{0}, \mathrm{~S}(0)=s_{0}, \mathrm{E}(0)=e_{0}, \mathrm{I}(0)=i_{0} \text { and } \\
& R(0)=r_{0}
\end{aligned}
$$

Where $D^{\alpha}=\frac{d^{\alpha}}{d t^{\alpha}}$ is the Caputo fractional derivative? Because model (1.3) monitors the dynamics of human populations, all the parameters are assumed to be nonnegative. Furthermore, it can be shown that all state variables of the model are nonnegative for all time $t \geq 0$. (See, for instance, $[27,28])$.

\section{BASIC DEFINITION:}

There are several definitions of a fractional derivative of order $\alpha>0$. .e.g .Riemann-Liouville, Caputo and Generalized Functions Approach. The most commonly used definitions are the Riemann-Liouville and Caputo. We give some basic definitions and properties of the fractional calculus theory which are used further in this paper.

Definition 1: The Riemann-Liouville fractional derivative operator $D_{R}^{\alpha}$ of order $\alpha$ is defined by

$m-1<\alpha<m$

$$
\alpha=m
$$

Theorem 2:

$$
F(k)=\sum_{k_{n-1}=0}^{k} \sum_{k_{n-2}=0}^{k_{n-1}} \ldots \sum_{k_{2}=0}^{k_{3}} \sum_{k_{1}=0}^{k_{2}} G_{1}\left(k_{1}\right) G_{2}\left(k_{2}-k_{1}\right) \ldots G_{n-1}\left(k_{n-1}-k_{n-2}\right) G_{n}\left(k_{n}-k_{n-1}\right)
$$

\section{Theorem 4:}

$$
\begin{aligned}
& \text { If } f(x)=\left(x-x_{0}\right)^{p}, \text { then } F(k)=\delta(k-\alpha p) \\
& \text { Where } \quad \delta(k)= \begin{cases}1 & \text { if } k=0 \\
0 & \text { if } k \neq 0\end{cases}
\end{aligned}
$$


Theorem 5:

If $f(x)=D_{x_{0}}^{q}[g(x)]$, then $F(k)=\frac{\Gamma(q+1+k / \alpha)}{\Gamma(1+k / \alpha)} G(K+\alpha q)$

Now let us expand the analytic and continuous function $f(x)$ in terms of fractional power series as follows:

$$
f(x)=\sum_{K=0}^{\infty} F(K)\left(\mathrm{x}-\mathrm{x}_{0}\right) K / \alpha
$$

$$
F(K)=\left\{\begin{array}{lc}
\text { if } K / \alpha \in Z^{+}, & \frac{1}{(K / \alpha) !}\left[\frac{{ }_{d} K / \alpha}{d x} K / \alpha\right) \\
f K / \alpha \notin Z^{+}, & 0 .
\end{array}\right.
$$

Where is the order of fractional differential considered?

\section{IMPROVEMENT CONVERGENT Equation (1.3) can be taking the form.}

\section{OF MSEIR EPIDEMIC MODEL:}

$$
\begin{aligned}
& D_{t}^{\alpha_{1}} m-(d+q)(\mathrm{e}+i+r)+\delta m=0 \\
& D_{t}^{\alpha_{1}} s-\beta s i+\delta m=0 \\
& D_{t}^{\alpha_{1}} e-\beta s i+(\varepsilon+d+q) \mathrm{e}=0 \\
& D_{t}^{\alpha_{1}} i-\varepsilon e+(\gamma+d+q) \mathrm{i}=0 \\
& D_{t}^{\alpha_{1}} r-\gamma i+(d+q) \mathrm{r}=0
\end{aligned}
$$

Then by using formula in the [18] then

$$
\begin{aligned}
& D^{\alpha} m=\sigma_{\alpha, k} \sum_{j=1}^{n} \omega_{j}^{(\alpha)}\left[m_{n-j+1}-m_{n-j}\right], D^{\alpha} s=\sigma_{\alpha, k} \sum_{j=1}^{n} \omega_{j}^{(\alpha)}\left[s_{n-j+1}-s_{n-j}\right], D^{\alpha} e=\sigma_{\alpha, k} \sum_{j=1}^{n} \omega_{j}^{(\alpha)}\left[e_{n-j+1}-e_{n-j}\right] \\
& D^{\alpha} i=\sigma_{\alpha, k} \sum_{j=1}^{n} \omega_{j}^{(\alpha)}\left[i_{n-j+1}-i_{n-j}\right] \text { And } D^{\alpha} r=\sigma_{\alpha, k} \sum_{j=1}^{n} \omega_{j}^{(\alpha)}\left[r_{n-j+1}-r_{n-j}\right]
\end{aligned}
$$

So by substitute in equation (1-1) we have got

$$
\begin{aligned}
& \sigma_{\alpha, k} \sum_{j=1}^{n} \omega_{j}^{(\alpha)}\left[m_{n-j+1}-m_{n-j}\right]-(d+q)\left(\mathrm{e}_{n}+i_{n}+r_{n}\right)+\delta m_{n}=0 \\
& \sigma_{\alpha, k} \sum_{j=1}^{n} \omega_{j}^{(\alpha)}\left[s_{n-j+1}-s_{n-j}\right]+\beta s_{n} i_{n}-\delta m_{n}=0 \\
& \sigma_{\alpha, k} \sum_{j=1}^{n} \omega_{j}^{(\alpha)}\left[e_{n-j+1}-e_{n-j}\right]-\beta s_{n} i_{n}+(\varepsilon+d+q) \mathrm{e}_{n}=0 \\
& \sigma_{\alpha, k} \sum_{j=1}^{n} \omega_{j}^{(\alpha)}\left[i_{n-j+1}-i_{n-j}\right]-\varepsilon e_{n}+(\gamma+d+q) \mathrm{i}_{n}=0 \\
& \sigma_{\alpha, k} \sum_{j=1}^{n} \omega_{j}^{(\alpha)}\left[r_{n-j+1}-r_{n-j}\right]-\gamma i_{n}+(d+q) \mathrm{r}_{n}=0
\end{aligned}
$$


By putting $\mathrm{n}=1,2, \ldots, \mathrm{n}$, we proved that $J_{1}, J_{2}, \ldots, \mathrm{J}_{n} \neq 0$

This means that MSEIR epidemic model is uniformly convergent

\section{SOLUTION OF MESIR EPIDEMIC} MODEL:

$$
\begin{aligned}
& M(K+1)=\frac{\Gamma\left(\alpha_{1} \mathrm{k}+1\right)}{\Gamma\left(\alpha_{1}(\mathrm{k}+1)+1\right)}\{(\mathrm{d}+\mathrm{q})[\mathrm{E}(\mathrm{K})+\mathrm{I}(\mathrm{K})+\mathrm{R}(\mathrm{K})]-\delta M(K)\} \\
& \mathrm{S}(K+1)=\frac{\Gamma\left(\alpha_{2} \mathrm{k}+1\right)}{\Gamma\left(\alpha_{2}(\mathrm{k}+1)+1\right)}\left\{-\beta \sum_{l=0}^{K} S(K) I(K-l)+\delta M(K)\right\} \\
& \mathrm{E}(K+1)=\frac{\Gamma\left(\alpha_{3} \mathrm{k}+1\right)}{\Gamma\left(\alpha_{3}(\mathrm{k}+1)+1\right)}\left\{\beta \sum_{l=0}^{K} S(K) I(K-l)-(\varepsilon+d+q) \mathrm{E}(K)\right\} \\
& \mathrm{I}(K+1)=\frac{\Gamma\left(\alpha_{4} \mathrm{k}+1\right)}{\Gamma\left(\alpha_{4}(\mathrm{k}+1)+1\right)}\{\varepsilon E(K)-(\gamma+d+q) \mathrm{I}(K)\} \\
& \mathrm{R}(K+1)=\frac{\Gamma\left(\alpha_{5} \mathrm{k}+1\right)}{\Gamma\left(\alpha_{5}(\mathrm{k}+1)+1\right)}\{\gamma I(K)-(d+q) \mathrm{R}(K)\}
\end{aligned}
$$

Also we use equation (3-2) to transform the initial condition.

So we have got the following relation.

$$
\begin{array}{ll}
M(\mathrm{~K})=0 & \text { for } K=1,2, \ldots, \alpha_{1} \beta_{1}-1 \\
S(K)=0 & \text { for } K=0,1,2, \ldots, \alpha_{2} \beta_{2}-1 \\
E(K)=0 & \text { for } K=0,1,2, \ldots, \alpha_{3} \beta_{3}-1 \\
I(K)=1 & \text { for } K=0 \\
I(K)=0 & \text { for } K=1,2, \ldots, \alpha_{4} \beta_{4}-1 \\
\mathrm{R}(K)=0 & \text { for } K=0,1,2, \ldots, \alpha_{5} \beta_{5}-1
\end{array}
$$

Therefore we take the value of $\alpha_{1}=\alpha_{2}=\alpha_{3}=\alpha_{4}=\alpha_{5}=1$

solution for MSEIR Model.

$m(t)=(d+q) t-\frac{1}{2}\left\{(d+q)^{2}+\delta(d+q)\right\} t^{2}+\frac{1}{6}\left\{2(d+q)(\gamma+d+q)^{2}+2(d+q)\left(\gamma^{2}+\delta\right)+2(d+q)^{2}(2 \gamma+\delta)\right\} t^{3}+\ldots$ $s(t)=\frac{1}{2} \delta(\mathrm{d}+\mathrm{q}) t^{2}+\frac{1}{6} \delta\left\{(d+q)^{2}+(d+q)(\beta+\delta)\right\} t^{3}+\ldots$

$e(\mathrm{t})=\frac{1}{6} \delta \beta(d+q) t^{3}+\ldots$

$\mathrm{i}(\mathrm{t})=1-(\gamma+d+q) \mathrm{t}+\frac{1}{2}(\gamma+d+q)^{2} t^{2}-\frac{1}{6}(\gamma+d+q)^{3} t^{3}+\ldots$

$r(t)=\gamma t-\frac{1}{2}\left\{\gamma^{2}+2 \gamma(d+q)\right\} t^{2}+\frac{1}{6}\left\{\gamma(\gamma+d+q)^{2}+(d+q) \gamma^{2}+2 \gamma(\mathrm{d}+\mathrm{q})^{2}\right\} t^{3}+\ldots$

Obviously table 1:

\begin{tabular}{|c|c|c|c|c|c|}
\hline $\mathbf{K}$ & $\mathrm{M}(\mathrm{K})$ & $\mathrm{S}(\mathrm{K})$ & $\mathrm{E}(\mathrm{K})$ & $\mathrm{I}(\mathrm{K})$ & $\mathrm{R}(\mathrm{K})$ \\
\hline $\mathbf{0}$ & $\mathbf{0}$ & $\mathbf{0}$ & $\mathbf{0}$ & $\mathbf{1}$ & $\mathbf{0}$ \\
\hline
\end{tabular}




\begin{tabular}{|c|c|c|c|c|c|}
\hline 1 & $(d+q)$ & $\mathbf{0}$ & $\mathbf{0}$ & $-(\gamma+d+q)$ & $\gamma$ \\
\hline 2 & $-2\left\{(d+q)^{2}+\delta(d+q)\right\}$ & $2 \delta(\mathrm{d}+\mathrm{q})$ & $\mathbf{0}$ & $2(\gamma+d+q)^{2}$ & $-2\left\{\gamma^{2}+2 \gamma(d+q)\right\}$ \\
\hline 3 & $3\left\{\begin{array}{l}2(d+q)(\gamma+d+q)^{2}+2(d+q) \\
\left(\gamma^{2}+\delta\right)+2(d+q)^{2}(2 \gamma+\delta)\end{array}\right\}$ & $6 \delta\left\{\begin{array}{l}(d+q)^{2}+ \\
(d+q)(\beta+\delta)\end{array}\right\}$ & $6 \delta \beta(d+q)$ & $-6(\gamma+d+q)^{3}$ & $6\left\{\begin{array}{l}\gamma(\gamma+d+q)^{2}+ \\
(d+q) \gamma^{2}+2 \gamma(\mathrm{d}+q)^{2}\end{array}\right.$ \\
\hline
\end{tabular}

\section{NUMERICAL SOLUTION:}

Also we can use fractional to obtain the solution of MSETR MODEL for fractional value.
We put $\mathrm{k}=0,1,2,3$, substituted in equations (4.2), (4.3), and substituted in equation (2.3), So we have got the following result

Let us take the value of $\alpha_{1}, \alpha_{2}, \alpha_{3}, \alpha_{4}, \alpha_{5}$ equal too 0.9

we will have got.

$$
\begin{aligned}
& m(t)=\frac{(d+q)}{\Gamma(19 l 10)} t-\frac{1}{\Gamma(28 / 10)}\left\{(d+q)^{2}+\delta(d+q)\right\} t^{2}+\frac{1}{\Gamma(37 / 10)}\left\{(d+q)(\gamma+d+q)^{2}+(d+q)\left(\gamma^{2}+\delta\right)+(d+q)^{2}(2 \gamma+\delta)\right\} t^{3}+\ldots \\
& s(t)=\frac{1}{\Gamma(28 / 10)} \delta(\mathrm{d}+\mathrm{q}) t^{2}+\frac{1}{\Gamma(37 / 10)} \delta\left\{(d+q)^{2}+(d+q)(\beta+\delta)\right\} t^{3}+\ldots \\
& e(\mathrm{t})=\frac{1}{\Gamma(37 / 10)} \delta \beta(d+q) t^{3}+\ldots \\
& \mathrm{i}(\mathrm{t})=1-\frac{(\gamma+d+q)}{\Gamma(19 l 10)} \mathrm{t}+\frac{1}{\Gamma(28 / 10)}(\gamma+d+q)^{2} t^{2}-\frac{1}{\Gamma(37 / 10)}(\gamma+d+q)^{3} t^{3}+\ldots \\
& r(t)=\frac{\gamma}{\Gamma(19 l 10)} t-\frac{1}{\Gamma(28 / 10)}\left\{\gamma^{2}+2 \gamma(d+q)\right\}^{2}+\frac{1}{\Gamma(37 / 10)}\left\{\gamma(\gamma+d+q)^{2}+(d+q) \gamma^{2}+2 \gamma(\mathrm{d}+\mathrm{q})^{2}\right\} t^{3}
\end{aligned}
$$

\begin{tabular}{|c|c|c|c|c|c|}
\hline $\mathbf{K}$ & $\mathrm{M}(\mathrm{K})$ & $S(K)$ & $\mathrm{E}(\mathrm{K})$ & $\mathrm{I}(\mathrm{K})$ & $\mathrm{R}(\mathrm{K})$ \\
\hline 0 & 0 & O & $\mathbf{0}$ & 1 & 0 \\
\hline 1 & $\frac{(d+q)}{\Gamma(19 l 10)}$ & 0 & 0 & $-\frac{(\gamma+d+q)}{\Gamma(19 l 10)}$ & $\frac{\gamma}{\Gamma(19 l 10)}$ \\
\hline 2 & $-\frac{1}{\Gamma(28 / 10)}\left\{(d+q)^{2}+\delta\right.$ & $\frac{1}{\Gamma(28 / 10)} \delta(\mathrm{d}+\mathrm{q})$ & 0 & $\frac{1}{\Gamma(28 / 10)}(\gamma+d+q)^{2}$ & $-\frac{1}{\Gamma(28 / 10)}\left\{\gamma^{2}+2 \gamma(d+q)\right\}$ \\
\hline 3 & $\frac{1}{\Gamma(37 / 10)}\left\{\begin{array}{l}(d+q)(\gamma+d+q)^{2}+ \\
(d+q)\left(\gamma^{2}+\delta\right)+ \\
(d+q)^{2}(2 \gamma+\delta)\end{array}\right\}$ & $\frac{1}{\Gamma(37 / 10)} \delta\left\{\begin{array}{l}(d+q)^{2}+ \\
(d+q)(\beta+\delta)\end{array}\right\}$ & $\frac{1}{\Gamma(37 / 10)} \delta \beta(d+q)$ & $-\frac{1}{\Gamma(37 / 10)}(\gamma+d+q)^{3}$ & $\frac{1}{\Gamma(37 / 10)}\left\{\begin{array}{l}\gamma(\gamma+d+q)^{2}+ \\
(d+q) \gamma^{2}+2 \gamma(\mathrm{d}+\mathrm{q})^{2}\end{array}\right\}$ \\
\hline
\end{tabular}

Obviously table 2: 


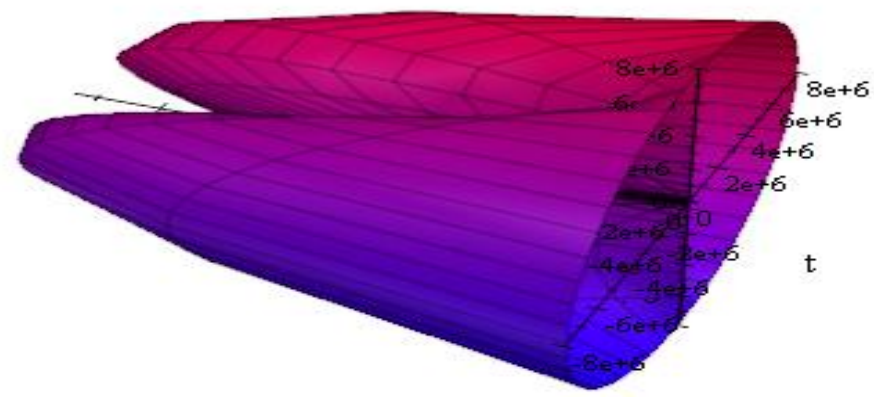

Figure1: (By using scientific work place programing) the behavior of the approximate solution ( $M(t), S(t), E(t), I(t)$ and $R(t)$ ) respectively, at $b=1, \beta=100, \mu=0.02, \zeta=1, \gamma=0.5, \sigma=0.05$ with initial condition
$\mathrm{M}(0)=0, S(0)=0, E(0)=0, I(0)=1$ and $R(0)=0$.

\section{CONCLUSIONS}

In this research has been to provide some definitions, which helped in solving the model sir through which study the effect of the vaccine on diseases such as Aids Caputo definition of fractional calculus, as well as the definition of the RiemannLiouville. Was also provided some scientific theories in ways to solve differential equations which fractional contributed significantly in solving the model sports sir., And also was the solution when fractional values to give an approximate solution for this model.

\section{REFERENCE}

[1] Chen,C.L. and Y.C. Liu, 1998. Differential transformation technique for steady nonlinear Heat conduction problems. Applied Mathematics and Computation, 95: 155-164.

[2] Chen, C.L. and Y.C. Liu, 1998. Solution of two point boundary value problems using the differential transformation method Journal of Optimization Theory and Applications, 99: 23-35.

[3] Chen, C.L., S.H. Lin and C.K. Chen, 1996. Application of Taylor transformation to Nonlinear predictive control problem. Applied Mathematical Modeling, 20: 699-710.

[4] Chen, C.K. and S.H. Ho, 1996. Application of differential transformation to eigenvalue Problems. Applied Mathematics and Computation, 79: 173-188.

[5] Howarth, L. On the Solution of the Laminar BoundaryLayer Equations. Proceedings of the Royal Society of London. 164:547-579( 1983)

[6] J.H. A coupling method of homotopy technique and perturbation technique for Nonlinear Problems, International Journal of Non-Linear Mechanics 3743(2000)

[7] J.H. Comparison of homotopy perturbation method and homotopy analysis method. Applied Mathematics and Computation.527-539(2004)

[8] J. H. Homotopy perturbation method: a new nonlinear analytical technique. Applied Mathematics and Computation.73- 79(2003)
[9] J.K. Zhou, Differential Transformation and Its Applications for Electrical Circuits, Huazhong University Press, Wuhan, China, 1986(in Chinese).

[10] N. Bildik, A. Konuralp, F. Bek, S. Kucukarslan, Solution of different type of the PDE by Differential transform method and A domain's decomposition method, Appl. Math. IComputer,172, 551-567(2006).

[11] I.Podlubny. Fractional differential equations. An introduction to fractional derivatives Fractional differential equations some methods of their Solution and some of their applications. Academic Press, San Diego, 1999.

[12] M.Caputo. Linear models of dissipation whose Q is almost frequency independent Part II. J Roy Austral Soc., 13:529-539, 1967.

[13] Mathematical Models in Biology by Leah EdelsteinKeshet, SIAM, 2005.

[14] "The Mathematics of Infectious Disease" by Herbert W. Hethcote, SIAM Review, Vol. 42, No. 4, p. 599-653, 2000.

[15] The Geographic Spread of Infectious Disease: Models and Applications by Lisa Sattenspiel With contributions by Alun Lloyd, Princeton, 2009.

[16] A.A.M.Hassan, S. H. H. Ibrahim, A.M. S. Mahdy and M. G. M.Ibrahim, "a new solution of sir model by using (DFTM)" Vol. 4, No. 11, April, 2014.

[17] V.S.Erturk and S.Momani,"solving systems of fractional differential equations using (DTM)" 215, (2008) 142-151

[18] N. H. Sweilam, M. M. Khader and A. M. S. Mahdy. "Crank-Nicolson Finite Difference Method For Solving Time-Fractional Diffusion Equation" Vol. 2. 2012, No. 2, pp. 1-9. ISSN: 2090-5858.

[19] Odibat Z. and S. Momani, A generalized differential transform method for linear partial Differential equations of fractional order, Applied Mathematics Letter, 21(2), 194-199, (2008).

[20] M.O. Ibrahim, I.I. Raji, A. Aladesuyi and A.Nwagwo "On the Homotopy analysis method of MSEIR epidemic 
model”, (IOSR-JAP) 2278-4861.Volume 6, Issue 3 Ver. II (May-Jun. 2014), PP 55-61

[21] A.A.M. Arafa, S.Z. Ridaand M. Khalil, (2012). Solutions of fractional or der model of Childhood diseases with constant vaccination strategy, Mathematical Sciences ,Letter 1(1),

[22] E.O.Ifidon,(2009)an Application of Homotopy Analysis to the Viscous Flow Past a Circular Cylinder. J.Appl. Math. 1-17.

[23] G.A.NgwaandW.Shu,(2000).A Mathematical model for endemic malaria with variable human And mosquito populations, Mathematical and Computer Modeling32:747-763.

[24] H.W.Hethcote, (2000). The Mathematics of Infectious Diseases, SIAM Review 42(4):599-653.
[25] L.Yajie,B.T. Nohara, and S.J. Liao,(2010). Series solutions of coupled Van- der Pol equation By means of homotopy analysis method, JournalofMathematicalPhysics51:1-12.

[26] L. J 'odar, R. J. Villanueva, A. J. Arenas, and G. C. Gonz'alez, "Nonstandard numerical Methods For a mathematical model for influenza disease," Mathematics and Computers in Simulation, Vol.79, no. 3, pp. 622633, 2008.

[27] E. H. Elbasha, C. N. Podder, and A. B. Gumel, "Analyzing the dynamics of an SIRS vaccination Model with waning natural and vaccine-induced immunity," Nonlinear Analysis, vol. 12, no. 5, pp. 2692 2705, 2011 